\title{
Energy, Environment and Climate: From the Past to the Future
}

\author{
Alberto Mirandola ${ }^{1 *}$ and Enrico Lorenzini ${ }^{2}$ \\ ${ }^{1}$ University of Padova, Dept. of Industrial Engineering, 1 Via Venezia, 35131 Padova, Italy \\ ${ }^{2}$ University of Bologna, Dept. of Industrial Engineering, 2 Viale Risorgimento, 40136 Bologna, Italy \\ Email: alberto.mirandola@unipd.it
}

\begin{abstract}
This paper provides some considerations on the energetic, environmental and climatic issues of the present time, starting from the knowledge of what has happened in the past. The paper does not intend to be exhaustive, since knowledge and culture, both in their broad meaning and in relation to the specific subject, are continuously developing and many certainties of today may inevitably be further discussed and even objected in the future. The authors agree on the need of gradually shifting from non-renewable to renewable energy sources, but also want to emphasize the valuable contribution given in the last two centuries by fossil fuels to the development of our society, underlining that their replacement cannot occur in short time: such an attempt would lead to shortage of resources for the increasing needs of a still growing world population. The difference between the environmental and the climatic issues will be remarked, highlighting the need to deepen their knowledge to better understand the related events and their causes: this step will be necessary to reliably forecast the future changes and ensure that we will be able to face them by proper actions in due time.

From a global prospective of the human society, the necessity to identify some priorities in carrying out suitable actions to tackle the problems of the present time will also be highlighted.
\end{abstract}

Keywords: Energy, Environment, Climate.

\section{INTRODUCTION}

Man's history on our planet developed according to the knowledge and to the social organization of people in the various areas and ages; but also the environmental conditions and the available resources, exploitable with proper technical abilities, had a great impact. The environment, in turn, was influenced by the climate, characterized by remarkable, sometimes drastic, changes during the history of the earth [12]. The climate, mainly determined by the dynamics of the solar system and other events occurring within the planet (volcanoes, earthquakes, etc.) [3-4-5], affected the events and the life on earth and water, forests and farming, with great impact on people's lives, their habits and thinking, their migrations, the wars. Our ancestors, being not able to deeply understand the natural events, generally believed they were determined by gods' intervention.

Of course, mankind did not passively accept the climate changes, but tried to modify the environment to better adapt it to their needs: for example, by knocking down trees, cultivating land, regulating flow of waters, building roads, villages and towns. Before the era of fossil fuels the availability of energy was scarce and the population increased very slowly because of the lack of resources. In the ancient Roman period the world population was about 200-300 million people and reached one billion only around half of the XIX century. Since then the population "exploded" together with the energy consumption: now we are about 7.3 billion (still increasing) and the annual energy use is about 14,000 Mtoe (million tons of oil equivalent), $80-85 \%$ of which supplied by fossil fuels [6-7].

This exponential growth was enabled by the use of all available energy sources: fossil fuels, uranium, biomasses, hydro-energy. Solar energy, under all its different forms, has always given a fundamental contribution to the maintenance of natural cycles (water, carbon, etc.) and the growth of forests, crops, grass for pastures; solar energy is now increasingly exploited by innovative systems (solar thermal and photovoltaic panels, windmills, etc.); but the contribution of these technologies is still low.

In the long (very long) time solar energy will probably be our future [8-9-10]; but now, and for many years to come, fossil fuels are and will be a fundamental resource for our life. Anyhow, our planet has a limited size: the present enormous number of humans and animals and the big quantity of activities in our society not only determines a relatively fast depletion of resources, but also an unprecedented environmental impact. Our generation must face fundamental problems: for how long will the nonrenewable resources be available? And how much time will be needed to replace them with renewables without depriving people from required resources with negative impact on the economy and the way of life in too short time? Could this trigger social conflicts and wars? How will the lifestyle of next generations change in the future? 
Another aspect has recently attracted the attention of politicians and scientists: climate changes. Indeed, this might turn out to be a false problem: climate has always changed in the history of our planet and it will keep changing also in the future. But now the question is: what are the key factors affecting climatic changes in the present time? How much do human activities influence the climate, since they are now much more intense than in the past? Will the climate changes mainly be determined by natural events as occurred in the past or by anthropogenic causes? If the present climatic events are mainly caused by humans and they are expected to be harmful, it would be of paramount importance to oppose them with appropriate measures; if not, adaptation to the changes would be more reasonable. Probably, both "mitigation" and "adaptation" will be necessary.

\section{ENERGY, ENVIRONMENT}

POPULATION AND

THE

Men's ability in exploiting the energy sources has determined the development of our civilization, with its positive and negative effects. The present society depends on non-renewable sources to a large extent: fossil fuels are still covering about $80-85 \%$ of total energy supply, as indicated earlier. These sources have modified and generally improved our life, enabling humanity to develop knowledge, science and technology. As a result, a large amount of food has been produced, comfort of buildings has improved, education has become available to an increasing percentage of population; the average duration of human life has increased thanks to the progress of medicine and hygiene; communication and transportation systems have enabled a growing number of people to travel to remote destinations and meet different people. In conclusion, the comfort of our lives has improved, enabling a better life for us. Therefore, it seems rather ungenerous to demonize fossil fuels, without which we would not be what we are.

But the limited size of the earth cannot allow current exponential growth of population and energy consumption to continue for a long time: the non-renewable sources are being depleted and the planet cannot recycle materials and energy beyond certain quantities. In conclusion, population, energy and environment are correlated with conflicting implications: a reasonable balance is needed among them and this need is occurring for the first time in our history.

These issues must be faced with the appropriate sense of reality: utopia and ideology often do not bring effective solutions. A gradual replacement of fossil fuels by renewables must be planned, but thinking it would happen in a short time frame is an illusion. Longer time is needed, for both the intrinsic characteristics of renewable sources and the expected deep social and economic transformations, which should not be sudden, to avoid economic shocks, social disorder and conflicts.

Will renewable sources be able to sustain a society with high degree of energy consumption? This is a crucial question, since these sources have the following negative characteristics which make their use rather expensive and problematic (while technology is rapidly improving the efficiency of these systems):

low power density, with corresponding need of large surface to tap solar energy and consequent large amount of materials required; discontinuous and unpredictable availability, with consequent relatively low degree of utilization and need for integrative generation and storage systems;

dependence on the characteristics of the installation site (latitude, elevation, average wind velocity, etc.).

Based on the previous considerations, it is advisable to address the scientific and technological research, which is the driver of progress, in the direction of further improving the use of both renewable sources and also traditional fossil fuels [11-12-13], with the target of employing them more effectively and efficiently (besides the first-law efficiency, also the second-law one must be assessed to rationally evaluate their exploitation).

On the other hand, this target has been continuously pursued in the whole history of energy use in the last century. An example is the technology of coal-fired plants: the overall efficiency, which was about $15 \%$ in the first decades of the $20^{\text {th }}$ century, is expected to reach $55 \%$ by 2020 . Also the environmental impact, which was not perceived and understood as a problem in the first decades of the last century, has been progressively reduced to very low values if compared with the previous ones. Some fundamental issues contributed to improve the technology of steam power plants:

the improvements of plant configuration and thermodynamic cycles, that led to continuously increasing values of pressure and temperature and, as a consequence, of the efficiency;

the design, manufacture and maintenance of the principal components (steam generators, turbines, condensers, etc.);

the treatment of water, fuels and flue gases;

the progress of the materials used in the most stressed machines and devices, whose more and more sophisticated composition and technology brought to mechanical performances that were not conceivable in the past;

- the monitoring and control systems, which enable the operators to continuously check and assess the working parameters and the overall performance;

the simulation techniques, which are a valuable help to compare the actual with the predicted performance of each component.

Analogous considerations can be done for the other fossil fuel technologies (gas turbines, reciprocating engines): the increase of efficiencies was reached together with a progressive reduction of the environmental impact. In addition, more operational flexibility was obtained to comply with the liberalization of the energy market.

But also the plants fed by renewable sources are continuously improving: while efficiencies increase, the costs are decreasing, bringing better competitiveness. Of course, the characteristics of the renewable sources, previously mentioned, require the development of new storage systems, which will be an important issue of research in the next years.

And we must not forget other innovative research topics: the use of low-enthalpy sources (geothermal energy) and biomasses combined with heat pumps and Organic Rankine Cycles; the very high efficiency of combined gas-steam plants and cogenerative systems; the hybrid and electric vehicles; the insulation techniques and the plants that increase the efficiency of buildings; etc.

Moreover, the organization of the conversion and utilization systems (transportation, industry, household equipment, etc.) will have a very important role.

Technology is and will be in progressive evolution. The political strategies should lead toward a society and a life 
style which will probably be quite different from the present ones; but this process ought to be very slow and gradual. The role of energetic education will be fundamental in order to get consensus when making decisions which may have unpopular implications [14]. Communication is also very important [15]: it is not appropriate to spread twisted information or hush up part of the truth to steer public opinion and influence attitudes and actions of a specific target audience, as frequently (unfortunately) done by politicians: transparency is always of paramount importance.

An example of incorrect communication is the information generally spread about the environmental pollution of our cities. Referring to the Italian plane of the Po river (Pianura Padana), we know that it is a critical area because of its geographical location: it is surrounded by mountains which make the atmospheric circulation rather difficult. Moreover, it is characterized by very large population density and widespread manufacturing activities. Therefore, the concentration of polluting substances cannot fall below certain levels, while this can be achieved in many other areas in Europe. As a consequence, the limits fixed by the European Union are often exceeded, in spite of the political measures. The mass media continuously highlight this situation and the related risks for public health.

Then people feel is that the environmental pollution is increasing, causing a continuous growth of inherent diseases and deaths. This feeling brings a permanent state of alarm and leads the public to exert pressure on the local authorities, who are pushed to take emergency measures that are generally useless. The atmospheric pollution is a fact; but what part of the truth is not mentioned?

First, the pollution across Europe was much higher some decades ago, when the efficiency of boilers, cars, trucks and power plants was lower and the abatement systems were almost inexistent. At that time the public perception of the pollution problems was scarce and no limits had been stated to the concentration of polluting substances. Over time, the increased awareness of these issues triggered an improvement of the energy systems and led the European authorities to define severe concentration limits. Therefore, the environmental situation significantly improved everywhere; but at this point it will be very difficult to further decrease these concentrations in the most critical areas, as the Po river valley. On the contrary, it is relatively easy to comply with the regulations in the areas having low density of population and where the weather is generally windy or rainy. Further improvements would be possible by changing the lifestyle and promoting scientific research on these topics, but a long time will be required. Different considerations can be done for some developing countries (China, India, etc.): these countries are much polluted now; they should not move along the same paths followed by the industrialized countries in the past, because now it is possible to act more rationally, even without giving up the economic development.

Second, the average duration of human life has increased very much in the last decades, mainly in the industrialized countries. In Italy, for example, life expectancy at the birth was 69 years in 1960, 85 years in 2015 (little more only in Japan). This means that public health also improved compared to 50 years ago, thanks to the progress in medicine, the improved hygiene, the people ambition towards a healthier lifestyle. If the number of diseases is increasing (cancer, illness of the respiratory apparatus, etc.), it depends on the old age of a significant part of the population: the old people more easily fall ill and, sooner or later, ... they will die!
In short, it is ethically inappropriate to spread incomplete information, manipulating part of the truth, to support one's ideas: the pollution must be opposed, but without rising unjustified alarm. The mass media are particularly prone to spread negative and tragic news, because they attract the public much more than positive ones.

The world energetic situation is evolving: the developed countries aim at gradually reducing their energy consumption through rational policies, management and treatment of waste, increase of efficiencies, improvement of organization, while developing countries are steeply increasing their energy use, causing a strong pressure on the resources and on the environment, also because of their very large population. Even though the growth rate of population is going to decrease, the effect on the overall world population will be significant after long time. In the meanwhile, the total energy need will increase for many years to come: non-renewable resources will still be necessary, but, of course, using them more and more efficiently will be advisable.

\section{THE CLIMATE CHANGES}

As already said before, the climate on the earth has always been changing. Considering these changes and trying to relate them to the human history and particularly to the European and Mediterranean history, we can roughly summarize the main climatic events of the last two millennia. Generally the relatively "warm" (interglacial) periods have been more favorable to humanity than the "cold" (glacial) ones: we can say that the human civilizations were the result of the interglacial periods, even though some climatic conditions might have been beneficial in some areas, while unfavorable in others.

Anyhow, historical and scientific precedents allow us to say that Europe and the Mediterranean area have experienced [1-2-16]:

3.1 A "Roman warm period" from about the III century B.C. and the IV A.D., when the Roman civilization reached its maximum power and strength;

3.2 A following "little glacial period" during the early Middle Ages (V-X century), when the severe climatic conditions caused a relative backward move and gave a remarkable contribution to migrations of peoples (Barbarian Invasions) and to the end of the Roman Empire, with its valuable culture and traditions;

3.3 An "interglacial medieval period" (XI-XIV century), which certainly had significant influence on the development of the Italian Renaissance;

3.4 A "little glacial age" (XV to half XIX century), during which the climate was generally cold and many historic and climatic negative events contributed to upset the European history;

3.5 The "interglacial period" of the present age (starting from the last decades of the XIX century); on average the temperature has been increasing, but with counter-trend periods: it decreased from 1945 to 1980 and has been relatively stable in the last 15 years. During the XX century the exponential growth of human population and the intense exploitation of energy sources has caused and is still causing a big impact on the environment.

Beyond these general trends, many anomalies occurred: unexpected warm and cold peaks, droughts, famine, floods, etc.). Some huge events were the consequence of very big volcanic phenomena. It is worth reminding the big explosion 
of Tambora volcano in 1815, which had a ravaging impact on a big part of the planet (and also a significant influence on the course and the strategies of the Waterloo battle, probably!). In fact, the following year 1816 is known as "the year without summer": in August, the temperatures fell down to about zero Celsius degrees in some areas of Europe and Northern America.

What can we say about the causes of these climatic events and of the present "warm" period? And what can we forecast for the future? In order to correctly interpret the climatic changes and their causes and forecast the future ones, it is necessary to start from the past and try to identify the phenomena that influenced and caused changes during the history of our planet [16-17]. For this purpose, we must use and combine knowledge from different research fields, since the science of climate is typically interdisciplinary.

Reliable instrumental measurements (temperature, humidity, etc.) are available for the last 100-150 years only. We must not believe that reliable models of the climate trends can be built on the basis of these data. Thinking that the climatic history of the last 150 years contains all the data that are necessary to understand the reasons of the present and future climate changes is unscientific. It is equivalent to believing that the last 150 years do not have a past and thinking that anthropic activities are the only causes of the global changes we are observing now (omnipotence attitude, indeed!) [17-18-19].

The environmental pollution of the last century is certainly due to the human activities, but the climate changes are a different matter and must be studied starting from previous climatic history. Synergy and collaboration are required between researchers of history and natural science and experts of instrumental records. Investigations, extended to the last millennia, about ancient geological sediments, archeological finds, historical archives and other documents, artistic creations, etc., matched and integrated with more recent instrumental data, can help interpret and understand the past and provide the baselines to forecast the next climatic and environmental changes and suggest suitable solutions to prepare our society to future conditions. The scientific disciplines related to the climatic issues are progressing; but the science of climate is young and still affected by many uncertainties. We should remember that 30-40 years ago, when the global temperature was decreasing, the scientists were prone to forecast a next new glacial age and suggested some actions that now seem to be naïve or even ridiculous. Then, after the inversion of the temperature in the 1980's, from the forecast of "Global Cooling" they have rapidly shifted to "Global Warming": how can we be sure of our certainties after these failures?

Many researches are being performed to understand the influence of the dynamics of the solar system on the past climate changes: variations of reciprocal positions of the sun, planets and satellites, precession and eccentricity of the earth orbit, axial tilt, etc. Now the anthropic activities are added to the natural causes and certainly have an influence; but the natural phenomena cannot be neglected. During the history of our planet interesting periodical oscillations [20-21-22], having different periods (for example, 10 years, 20 years, 60 years, 1000 years and others) have been observed: they interact with each other and contribute to complicate the main trends. The causes of these periodical changes must be deeply studied.

The global circulation models identified, collected and referred to in the IPCC reports, which ascribe the climatic changes to the human activity for about $97 \%$, do not consider all these phenomena. If applied to some specific period and compared with instrumental data available for that period, such models have generally failed in supplying reliable representation of the climate trends: they probably overestimate the anthropic influence, because are prone to undervalue the contribution of natural causes. Is it realistic to state that these natural causes, which determined the climate evolution during the whole history of our planet, have now become irrelevant due to the increased influence of anthropic civilization? Perhaps in the future, after careful investigations of the dynamics of the solar system and further collection of instrumental data, some belief will be reconsidered.

\section{OPEN PROBLEMS FOR THE MEDIUM AND LONG TERM}

Humanity, during its long history, have faced many difficult and upsetting circumstances and solved a lot of problems to survive, grow and improve its way of living in the planet. The present society lives in a crowded world that has become rather small if we consider the continuous population growth, the progressive exploitation of resources, the huge environmental problems. These problems are interdependent with each other and mixed with other very urgent matters. It will not be possible to solve all of them simultaneously: suitable priorities must be identified.

Climate changes will probably not be the first priority, despite what many opinion leaders may state. The mitigation of the environmental impact is, of course, necessary to improve the quality of our lives. Gradual decreasing the use of fossil fuels and greater attention to energy saving, without depriving people from the indispensable resources, is one of the priorities. But we must not forget that the climate will probably change anyway, regardless from what actions we undertake: it is important to be prepared, not to be caught by surprise. This means that not only "mitigation", but also "adaptation" is of paramount importance.

Anyhow, many other problems represent urgent priorities.

4.1) The rate of growth of world population should decrease, up to reach and stabilize an overall number of people consistent with the available resources. It is true that research and technology can make available some kinds of resources that now cannot be exploited; this issue occurred many times during our history. But, in spite of this consideration, a limit is given by the size and the overall recycling capacity of our planet. The road will probably be long; but we must take these aspects into account in setting and developing our strategies.

4.2) An acceptable level of education should be reached in the underdeveloped areas, to avoid that a large number of inhabitants become uncritical masses manipulated by the power of politics, economy, religion, ideology.

4.3) Excessive economic disparities among peoples are often origin of conflicts: the governments should act in order to reduce these disparities.

4.4) The resources of the earth are not infinite. New discoveries and new technologies will probably increase the reserves of materials and energy sources; but, of course, this process cannot continue indefinitely. Consequently, it is very important to preserve the resources of our planet. The idea of trying to populate other planets might be theoretically attractive, but we should better look after our old Mother Earth! 
4.5) Under the pressure of the growing population and the interests of business organizations, we have allowed many areas to become overcrowded and we have intensely built roads, bridges, buildings, factories, cities, etc., even in unadvisable locations, often neglecting the inherent risks. Wide areas have been savagely deforested, while some others, formerly cultivated, have been abandoned, letting forests to grow in complete disorder; the maintenance of critical territories has been neglected. Nature does not forgive unaware or irrational behavior: the climate and the weather can suddenly change, the natural events can be violent and it is much more reasonable to properly act in advance rather than putting in place emergency measures after calamities.

4.6) Coming back to education, it is also important to explain the prominence of the previous problems to the public. Education should lead people to behave ethically in their everyday life, learning to correctly interact with the others, independently of their traditions, ideologies, religions. In the Western Society a very important relatively recent conquest, allowed by education, is the concept of parity of rights and dignity of each person, man or woman. This conquest should gradually reach all the world, even considering that each civilization should preserve its culture, without imposing it to other peoples. This issue takes us to speak about terrorism, which, unfortunately, is a very serious and upsetting problem being experienced by our society in the present years. The religious fundamentalism brings terrorism; but the roots of terrorism are also to be looked for within uneducated, unaware, underdeveloped masses of people. Once more, education and freedom are the keys for a reasonable answer to this problem: educated people can be driven and encouraged to engage themselves in improving their life standard without trying to get unlikely benefits by violent actions.

4.7) What about the scientific and the technological research? It has been the driver for our progress during the whole history of humanity and should always be considered a priority, without forgetting that it should always be linked and matched with moral conscience and ethical thinking.

\section{CONCLUSIONS}

The interaction among energy, economy and the environment is the basis of our life. The availability of big amounts of energy drives our activities, which, in their turn, have big impact on the environment and on the economy. The human trend throughout the history has been influenced by man's ability in exploiting the natural resources; the steep growth of the world population in the last two centuries has been mainly due to the use of fossil fuels, which still contribute to the total energy consumption by $80-85 \%$. But the reserves of fossil fuels are not unlimited and, moreover, their use causes pollution and health problems.

Hence it is of fundamental importance to reorganize the energy systems; but we must consider that the world population of over 7 billion people (still increasing) needs a large amount of energy. The transition to a different balance between resources (from fossil fuels to renewables) is an epochal issue and will need a long time to be addressed, to avoid social conflicts and wars caused by shortage of the usual means of subsistence. It is a big challenge, which requires the support of science and technology, but also the development of a common perception on these problems.
Another important matter is the climatic situation and its connection with the environmental issues. The climate has always changed in the human history and beforehand: a superposition of long time trends, periodical oscillations and sudden events has been observed. It is very difficult to forecast the future climate. Science and technology can help doing it, but it is necessary to assess these changes considering the historical background, matching the instrumental measurements, only available for the last 100150 years, with other "proxy" data supplied by scientific (and also humanistic) interdisciplinary researches.

The environmental status and climate are different issues and must not be mixed with each other. It is obvious that the environmental pollution is mainly caused by human actions; but how much do they impact on climate changes? It is not believable that the natural events, which have driven the whole history of climate up to the XIX century, have become irrelevant compared with the increased impact of human activities. The science of climate is very young; we should be humble and realize that many things are still to be discovered. Remembering that $30-40$ years ago the scientists shifted from the forecast of Global Cooling to that of Global Warming, it is very difficult to have true "certainties" about climate.

We must start from the past and deepen our knowledge on the history of climate to correctly interpret it and try to set the basis for a reliable forecast of the future. Climate changes are a big and complex issue, but perhaps we have more urgent priorities, connected to human conscience, moral behavior and personal ethics. Some of them have been outlined in the previous section: they require wisdom, honesty, grandstand and long-term view, decision-making attitude. Will humanity be able to take decisions and behave in the right way?

\section{REFERENCES}

[1] Wolfgang Behringer, A Cultural History of Climate, John Wiley \& Sons, ISBN100745645291, (also available in German and Italian), 2009.

[2] Guidoboni E., Navarra A. and Boschi E. Nella spirale del Clima. Culture e società mediterranee di fronte ai cambiamenti climatici, BUP -Bononia University Press (in Italian), 2008.

[3] obock A., Volcanic eruptions and climate. Reviews of Geophysics 38 (2), 191-219, 2000.

[4] Robock A., Climatic impact of volcanic eruptions. In H. Sigurdsson ed. Encyclopedia of Volcanoes, Elsevier, 935-942, 2015.

[5] Tolstoy M., Mid-ocean ridge eruptions as a climate valve. Geophysical Research Letters, 2015. DOI: 10.1002/2014GL063015.

[6] IEA (International Energy Agency), World Energy Outlook 2015.

[7] European Commission, 2015. EU Energy Figures: Statistical Pocketbook 2015.

[8] IPCC Assessment Reports: AR1, 1990; AR2, 1995; AR3, 2001; AR4, 2007; AR5, 2014.

[9] ENEA, 2015. Transition and Global Challenges towards Low Carbon Societies, Special Report.

[10] Lorenzini, E., 2006. Dalle cronache quotidiane il dramma italiano dell'energia. Pitagora, Bologna, Vol. III (in Italian).

[11] Mirandola, A., Stoppato, A. and Benato, A., 2015. Steam Power Generation; Handbook of Clean Energy 
Systems, John Wiley \& Sons, Ltd., Vol. 2 of 6 , pp.745-762, ISBN 978-1-118-38858-7.

[12] Stoppato, A., Mirandola, A. and Benato, A., 2015. Gas Turbines and Combined Cycle Gas Turbines; Compendium of Energy Science and Technology (in 10 Vols), Vol. 9, Stadium Press LLC, USA.

[13] Stoppato, A., Mirandola, A., Meneghetti, G. and Lo Casto, E., 2012. On the Operation Strategy of Steam Power Plants working at variable load: Technical and Economic Issues; Energy, the International Journal, Vol 37, pp.228-236, 2012.

[14] Ponchio, A. and Mirandola, A., "Professional ethics, engineering and energetic issues," American Journal of Energy Engineering, Science Publishing Group, vol. 2, issue 4, July 2014, pp. 87-95, 2014.

[15] Marin, F. and Mirandola, A., "Ethics, communication and propaganda about energetic and environmental topics." American Journal of Energy Engineering, ISSN 2329-1648, Sept. 20, DOI: 10.11648/j.ajee.

[16] Camuffo, D., Bertolin, C., Schenal, P., Craievich, A. and Granziero, R., "The Little Ice Age in Italy from documentary proxies and early instrumental records," Journal of Mediterranean Geography, vol. 122, pp. 17-20, 2014.
[17] NIPCC. Climate Change Reconsidered, CCR Reports 2009, 2011, 2013, 2014, 2015.

[18] Vai G., 2015. Tempo umano e tempo geologico: $i$ cambiamenti climatici sono un capro espiatorio? in "Prevedibile /Imprevedibile. Eventi estremi nel prossimo futuro" a cura di E.Guidoboni, F.Mulargia e V.Teti, Rubbettino, pp. 31-52 (in Italian).

[19] Rorsch, A., Ziegler, P. and Singer, S., "Inconsistency of modeled and observed tropical temperature trends," Energy and Environment, 2013.

[20] Scafetta, N. and Mazzarella, A., "The Arctic and Antarctic sea-ice area index records versus measured and modeled temperature data." Advances in Meteorology, vol. 2015, Article ID 481834, 8 pages, 2015. DOI: $10.1155 / 2015 / 481834$.

[21] Scafetta, N., "Discussion on climate oscillations: CMIP5 general circulation models versus a semiempirical harmonic model based on astronomical cycles," Earth-Science-Reviews, vol. 126, pp. 321357, 2013. DOI: 10.1016/j.earscirev.2013.08.008.

[22] Scafetta, N., "Discussion on the spectral coherence between planetary, solar and climate oscillations: a reply to some critiques." Astrophysics and Space Science, vol. 354, pp. 275-299, 2014. DOI: 10.1007/s10509-014-2111-8. 